\title{
Cinesioterapia no tratamento da Incontinência Urinária em mulheres idosas
}

Kinesiotherapy on treatment of Urinary Incontinences in elderly women

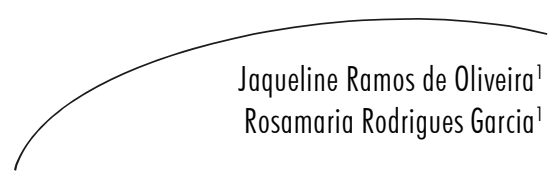

Resumo

A pesquisa tem como objetivo verificar o efeito da cinesioterapia sobre a perda de urina diária, alívio dos sinais e sintomas, e verificar o impacto da cinesioterapia na qualidade de vida das idosas com incontinência urinária. Estudo de intervenção com idosas com queixa de incontinência urinária, do Instituto Paulista de Geriatria e Gerontologia "José Ermírio de Moraes". Foram realizadas antes e após o tratamento, uma avaliação fisioterapêutica uroginecológica; aplicado o questionário de qualidade de vida Kings Health Questionnaire; e atendimento em grupo, com sessões semanais composto de exercícios para a musculatura do assoalho pélvico, por um período de três meses. A análise estatística dos dados foi realizada através do teste de Wilcoxon, comparando os dados antes e após o tratamento. O grupo estudado foi composto por 11 idosas, média etária de 74,2 anos, índice de massa corpórea média de $29,69 \mathrm{Kg} / \mathrm{m}^{2}$. A maioria (6) das pacientes apresentou incontinência urinária mista. O tempo médio da queixa de incontinência urinária foi de seis anos. A média do número de partos normais foi 5,09 . Observou-se redução na média de frequência de miç̧ões noturnas na presença de noctúria ( 3 versus 1,5$)$ e na média do número de situações de perda urinária aos esforços $(3,72$ versus 1,45$)$. Considerando o questionário de qualidade de vida, observou-se redução significativa nas médias e medianas dos escores em quase todos os domínios. Conclui-se que a cinesioterapia do assoalho pélvico foi positiva para obter melhoras sobre a perda de urina diária e alívio dos sinais e sintomas, bem como na qualidade de vida.

\section{Abstract}

This research aims to verify the kinesiotherapy's effects on daily urine loss, signs and symptoms relief, and to verify the kinesiotherapy's impact on the quality of life of UI (Urinary Incontinence) affected elderly women. Intervention study on aged women's UI complaints, from the "José Ermírio de Moraes" Paulista Geriatrics and Gerontology Institute. Before and after the treatment, an

Palavras-chave: Saúde do idoso. Qualidade de vida. Envelhecimento. Idoso. Incontinência Urinária. Terapia.

\footnotetext{
Instituto Paulista de Geriatria e Gerontologia "José Ermírio de Moraes”. Secretaria de Estado da
} Saúde. São Paulo, SP, Brasil. 
urogynecologic physiotherapeutic evaluation was conducted, and the quality of life "Kings Health Questionnaire" was administered. Group assistance, in weekly sessions composed of exercises for the pelvic floor muscularity, for a period of three months. The statistical analysis was performed through the Wilcoxon Test, comparing the before and after treatment data. The studied group was composed by 11 elderly women, mean age 74.2 years, mean body mass index of $29.69 \mathrm{~kg} / \mathrm{m}^{2}$. Most (six) of the patients presented MUI (mixed urinary incontinence). The mean UI complaint period was six years. The mean number of natural childbirths was 5.09. A decrease in the frequency of night urination was observed when nocturia was present (1.5 compared to 3), as well as in the mean number of urine loss situations due to effort (1.45 compared to 3.72). Considering the quality of life questionnaire, a significant decrease was observed in the scores' means and medians in almost all domains. It was concluded the pelvic floor kinesiotherapy was positive regarding urine loss, signs and symptoms relief, as well as quality of life.
Key words: Health of the Elderly. Quality of Life. Aging. Aged. Urinary Incontinence. Therapeutics.

\section{INTRODUÇÃO}

A International Continence Society (ICS) define incontinência urinária (IU) como queixa de qualquer perda involuntária de urina. ${ }^{1} \mathrm{~A}$ IU entre idosos é um achado comum e é muitas vezes erroneamente interpretada como parte natural do envelhecimento. Alterações que comprometem o convívio social como constrangimentos, perda da autoestima, depressão e isolamento, frequentemente fazem parte do quadro clínico, implicando problemas psicológicos e sociais para os pacientes e familiares. ${ }^{2-4}$

Embora possa ocorrer em todas as faixas etárias, a incidência da incontinência urinária aumenta com o decorrer da idade. Calcula-se que oito a $34 \%$ das pessoas acima de 65 anos possuam algum grau de incontinência urinária, sendo mais prevalente em mulheres. Aproximadamente 10,7\% das mulheres brasileiras procuram atendimento ginecológico queixando-se de perda urinária. ${ }^{5,6}$

Uma estimativa recente sobre custos diretos anuais relacionados à incontinência urinária em todas as idades apresenta valores que giram em torno de 16 bilhões de dólares nos Estados Unidos, sendo maior do que os custos diretos anuais para o câncer de mama, ovário, útero e colo de útero combinados. ${ }^{7}$

De acordo com Subak et al., ${ }^{8}$ além dos custos econômicos, a IU afeta substancialmente a qualidade de vida. É necessário que o tratamento seja efetivo, a fim de gerar diminuição dos gastos e melhora da qualidade de vida da paciente.

A IU determina repercussões importantes nos aspectos físicos, mentais e sociais das mulheres incontinentes. Quanto às repercussões sociais, a IU impede a mulher de sair de casa, ir a festas e ao clube, fazer viagens longas, frequentar a igreja e participar de atividades físicas, como caminhar, correr, jogar e dançar. Pode ter como consequência o afastamento social do indivíduo pelo constrangimento causado por esta doença., ${ }^{9}, 10$

Entre as principais consequências relacionadas à saúde física, destacam-se: a insuficiência renal, infecção do trato urinário, sepsemia, aumento do risco de quedas e fraturas, maceração da pele e formação de feridas, fator higiênico além da interferência na vida sexual, nas tarefas domésticas e no trabalho. ${ }^{6}$ A IU também pode estar relacionada a alterações no âmbito da saúde mental, através da depressão e perda da autoestima. Além disso, interfere negativamente, na qualidade de vida de muitas delas. ${ }^{9}, 10$

Para que não ocorra incontinência urinária, é necessário que haja um funcionamento adequado do aparelho urinário inferior, que implica integridade anatômica e dos centros e vias nervosas, que coordenam a ação da musculatura lisa e estriada do aparelho urinário e do pavimento pélvico. ${ }^{11}$ 
Dentre os vários tipos de IU, destacam-se: a incontinência urinária de esforço (IUE), a urgeincontinência ou bexiga hiperativa $(\mathrm{BH})$ e a incontinência urinária mista (IUM), caracterizada pela incontinência urinária de esforço associada à bexiga hiperativa. ${ }^{12}$

Segundo a ICS, a IUE é caracterizada pela perda involuntária de urina durante o esforço, por meio de exercícios físicos, espirro ou tosse, ou seja, ocorre quando está associada a qualquer atividade que aumente a pressão intra-abdominal. Ocorre devido a uma deficiência no suporte vesical e uretral que é feito pelos músculos do assoalho pélvico e/ou por uma fraqueza ou lesão do esfíncter uretral. ${ }^{1}$

Em 2002, a ICS redefiniu a bexiga hiperativa $(\mathrm{BH})$ como uma síndrome de urgência, com ou sem urge-incontinência (perda urinária involuntária acompanhada ou imediatamente precedida de urgência), frequentemente associada com o aumento da frequência urinária e, não de forma obrigatória, à presença de noctúria. Esta definição pode ser utilizada caso não haja quadro de infecção ou outras patologias associadas. A ICS ainda caracteriza a $\mathrm{BH}$ pela presença de contrações involuntárias do músculo detrusor, espontâneas ou provocadas, durante o período de enchimento vesical. ${ }^{1}$

\section{TRATAMENTO FISIOTERAPÊUTICO DA IU}

A fisioterapia, como forma abrangente de tratamento, visa a prevenção e tratamento curativo da IU por meio da educação da função miccional, informação a respeito do uso adequado da musculatura do assoalho pélvico, bem como o aprendizado de técnicas e exercícios para aquisição do fortalecimento muscular. ${ }^{13}$ São objetivos principais da fisioterapia a reeducação $d a$ musculatura do assoalho pélvico e seu fortalecimento, visto que, na maioria dos tipos de incontinência urinária, está presente uma redução da força desta musculatura. ${ }^{14}$

A reabilitação do trato urinário inferior (TUI) é definida como terapêutica não cirúrgica e não- farmacológica para restabelecimento da função adequada do TUI. ${ }^{1}$ Entre as principais modalidades de tratamento fisioterapêutico da incontinência urinária, encontram-se: o biofeedback, mudanças comportamentais, a eletroestimulação neuromuscular, os cones vaginais e a cinesioterapia. ${ }^{15}$

O biofeedback é um aparelho cuja técnica possibilita que a informação sobre o processo normal, fisiológico e inconsciente da contração muscular do assoalho pélvico, seja introduzida ao paciente e/ou ao terapeuta como sinal visual, auditivo ou tátil. ${ }^{1}$

A modificação comportamental é definida como a análise e alteração do relacionamento entre os sintomas da paciente e o seu ambiente, com o objetivo de tratar os modelos de micção inadequados ou mal adaptados. Estas modificações podem ser obtidas por modificação comportamental da paciente ou do ambiente em que ela vive. ${ }^{1}$

A eletroestimulação neuromuscular (EENM) é a aplicação de corrente elétrica que estimula a inervação da víscera pélvica ou o suprimento de sua inervação. O objetivo da EENM é induzir diretamente uma resposta terapêutica ou passar a modular as disfunções do TUI, intestinais e sexuais. ${ }^{1}$

Os cones vaginais foram desenvolvidos por Plevnik em 1985. Ele demonstrou que a mulher pode melhorar o tônus da musculatura pélvica introduzindo na cavidade vaginal cones de material sintético, exercitando a musculatura do períneo na tentativa de reter os cones e aumentando progressivamente o peso dos mesmos. $^{16}$

A cinesioterapia do assoalho pélvico compreende basicamente na realização dos exercícios de Kegel, que objetiva trabalhar a musculatura perineal para o tratamento da hipotonia do assoalho pélvico. Kegel ${ }^{17}$ e Kegel \& Powel $^{18}$ foram os primeiros pesquisadores nos Estados Unidos a prescrever exercícios específicos para o fortalecimento dos músculos do assoalho 
pélvico. $\mathrm{O}$ objetivo básico dos exercícios para fortalecimento da musculatura pélvica é o reforço da resistência uretral e a melhora dos elementos de sustentação dos órgãos pélvicos. ${ }^{19}$

O fortalecimento dos músculos do assoalho pélvico através da reeducação perineal tem-se revelado apropriada numa série de mulheres com incontinência urinária, constituindo a base da terapêutica conservadora. ${ }^{11}$

Visto que a população idosa vem aumentando significativamente ao longo do tempo e isto predispõe a um aumento da prevalência da IU, percebe-se a importância do tratamento conservador. Assim, emergiram os objetivos norteadores desta pesquisa, que são verificar o efeito da cinesioterapia sobre a perda de urina diária e o alívio dos sinais e sintomas referidos, bem como avaliar o impacto da cinesioterapia na qualidade de vida das idosas portadoras de IU.

\section{METODOLOGIA}

Trata-se de estudo de intervenção, prospectivo, realizado no Instituto Paulista de Geriatria e Gerontologia “José Ermírio de Moraes”, no período de maio a dezembro de 2009.

A população de estudo foi constituída por idosas (60 anos ou mais de idade) com queixa de IU que consigam locomover-se ao serviço para participar do programa, triadas da lista de espera do Setor de Fisioterapia do referido instituto, que concordaram em participar do estudo e assinaram espontaneamente o termo de consentimento livre e esclarecido. Foram excluídas idosas portadoras de tumores pélvicos, com cirurgia pélvica ou abdominal há seis meses, em vigência de infecção urinária, que fazem uso de medicação anticolinérgica, que já realizaram qualquer tipo de tratamento para IU, incapacidade de locomoção ao serviço e de compreensão ou déficit cognitivo, idosas com bexiga neurogênica e idosas que abandonaram o tratamento.

A coleta de dados foi realizada mediante avaliação fisioterapêutica e da qualidade de vida pré e pós-tratamento, de forma individual. A avaliação fisioterapêutica foi realizada através $\mathrm{da}$ história clínica realizada de forma detalhada, objetivando a coleta de dados relevantes que caracterizam a IU. Também foram consideradas informações referentes a antecedentes cirúrgicos, obstétricos e uso de drogas que pudessem comprometer a função do trato urinário inferior.

A avaliação da qualidade de vida foi realizada através do questionário King's Health Questionnaire (KHQ). Esse questionário avalia a qualidade de vida de pacientes com incontinência urinária. É constituído de 21 questões, divididas em oito domínios: percepção geral de saúde, impacto da IU, limitações da atividade diária, limitações físicas, limitações sociais, relações pessoais, emoções, sono/disposição.

Além desses parâmetros, existem duas escalas independentes. A primeira avalia a gravidade $\mathrm{da}$ IU, denominada medida de gravidade. A segunda considera a presença e a intensidade dos sintomas urinários. A pontuação é dada em cada um dos seus domínios, não havendo, portanto, pontuação geral. Os valores variam de $\mathrm{O}$ a 100 e, quanto maior a pontuação, pior é a qualidade de vida referente àquele critério.

A versão para o idioma português do questionário para avaliação da qualidade de vida, o KHQ, em pacientes portadoras de IU já validada por Tamanini et al., ${ }^{20}$ em 2003, mostrou-se confiável e válida.

As pacientes foram atendidas em grupo, com sessões uma vez por semana, compostas de exercícios específicos para a musculatura do assoalho pélvico, realizados nas posições sentada, deitada, de pé e andando, com duração de 30 minutos cada sessão. Inicialmente solicitou-se que sustentassem a contração do assoalho pélvico por cinco segundos, com um tempo de relaxamento de cinco segundos, de acordo com a tolerância da paciente. Foram formados dois grupos: Grupo GI, composto inicialmente por 13 pacientes atendidas no período de maio a julho de 2009. E Grupo GII, composto inicialmente por 12 pacientes atendidas no período de agosto a 
outubro de 2009. A média de sessões para os dois grupos foi de dez. As pacientes eram orientadas a realizarem os exercícios em casa.

Destes grupos, 14 idosas foram excluídas, sendo sete do grupo GI e sete do grupo GII, após avaliação completa e início do tratamento, por abandonarem o mesmo.

No primeiro encontro, foi realizada uma aula em PowerPoint sobre a definição da IU, seus sintomas, anatomia básica do assoalho pélvico, para esclarecimentos às pacientes a respeito da patologia e com o objetivo de promover a conscientização da contração da musculatura do assoalho pélvico.

Para a análise dos resultados, foi realizada uma comparação dos dados obtidos a partir da ficha de avaliação aplicados antes e no final do período de tratamento com a cinesioterapia e aplicado o teste de Wilcoxon, ${ }^{21}$ para comparar os escores do KHQ nos períodos antes e após tratamento. Fixou-se em 0,05 ou $5 \%$ o nível de rejeição da hipótese de nulidade.

O presente estudo obteve a aprovação do Comitê de Ética em Pesquisa do Instituto de Saúde, sob o parecer de número 006/2009.

\section{RESULTADOS}

O grupo estudado foi composto por 11 idosas, com idades de 65 a 83 anos, com média etária de
74,2 anos e IMC entre 22,3 e 49,3 com média de $29,69 \mathrm{Kg} / \mathrm{m}^{2}$. O tempo médio da queixa de IU variou de seis meses a mais de dez anos, com média de seis anos. Em relação aos antecedentes obstétricos, oito pacientes tiveram entre um e cinco partos e três tiveram nove ou mais partos. Quanto à via de parto, todas as pacientes tiveram no mínimo um parto vaginal e apenas duas tiveram também parto cesárea. A média do número de partos normais foi de 5,09.

Considerando o diagnóstico referente aos tipos de IU, a partir dos sintomas referidos observou-se que três pacientes obtiveram o diagnóstico de $\mathrm{BH}$, duas IUE e seis IUM antes do tratamento. Após o tratamento, três pacientes não referiam sintomas da IU.

Ao analisar a frequência de micções noturnas na presença de noctúria antes e após o tratamento, pelo teste de Wilcoxon, observou-se redução na média de 3 para $1,5(p=0,02)$, evidenciando uma redução significativa na frequência de micções noturnas.

Foram analisadas, antes e após o tratamento, as seguintes situações de perdas urinárias aos esforços relatadas na avaliação fisioterapêutica: espirrar, tossir, saltar, correr, rir, levantar peso, mudar de posição, levantarse pela manhã e subir escadas. Antes do tratamento, a média do número de situações de perda urinária aos esforços foi igual a 3,72. Após o tratamento, a média foi de 1,45 , como descrito na tabela 1 .

Tabela 1 - Pacientes segundo número de situações de perdas urinárias aos esforços, relatadas na avaliação fisioterapêutica, nos períodos pré e pós-tratamento. São Paulo, SP, 2009.

\begin{tabular}{cccc}
\hline Pacientes & Pré & Pós \\
\hline 1 & 2 & 1 \\
2 & 4 & 0 \\
3 & 2 & 1 \\
4 & 0 & 0 \\
5 & 6 & 0 \\
6 & 4 & 2 \\
7 & 5 & 3 \\
8 & 2 & 1 \\
9 & 9 & 5 \\
10 & 4 & 3 \\
11 & 3 & 0 \\
\hline Média & 3,72 & 1,45 \\
\hline
\end{tabular}


Ao analisar os dados referentes à retenção diante do primeiro desejo forte de urinar, antes do tratamento, dez pacientes referiam retenção com dificuldade e com perda involuntária de urina e uma não referiu dificuldade e conseguiu chegar seca ao banheiro. Após o tratamento, oito pacientes referiram retenção com dificuldade, sendo que quatro relataram perda involuntária de urina e quatro não perderam urina; e três pacientes não referiram dificuldade de retenção.

Com relação ao número de absorventes diários utilizados, antes do tratamento, cinco pacientes referiam utilizar de 1 a 3 absorventes diários e uma paciente, de 4 a 6 absorventes. Após o tratamento, as seis pacientes que utilizavam proteção, referiram a troca de 1 a 3 absorventes diários. As demais pacientes referiam não utilizar proteção alguma.

Como descrito na tabela 2, analisando-se os dados do questionário de qualidade de vida Kings Health Questionnaire (KHQ) antes e após o tratamento, pelo teste de Wilcoxon observou-se diminuição significativa nas médias e medianas dos escores nos domínios percepção geral da saúde, impacto da IU, limitação nas atividades de vida diária, limitações físicas, limitações sociais, emoções, sono e disposição, medidas de gravidade e escala de sintomas. O único escore que não apresentou diferença foi o relacionado a relações pessoais, pois nove pacientes referiram ausência de relação sexual e duas referiram não interferir.

Tabela 2 - Valores dos escores dos domínios do KHQ antes e após a cinesioterapia. São Paulo, SP, 2009.

\begin{tabular}{|c|c|c|c|c|c|}
\hline \multirow[t]{3}{*}{ Domínio } & \multicolumn{5}{|c|}{ KHQ } \\
\hline & \multicolumn{2}{|c|}{ Pré } & \multicolumn{2}{|c|}{ Pós } & \multirow[b]{2}{*}{$\mathrm{P} *$} \\
\hline & Média & Mediana & Média & Mediana & \\
\hline Percepção geral da saúde & 65,9 & 75,0 & 36,3 & 25,0 & 0,006 \\
\hline Impacto da IU & 63,6 & 66,6 & 48,5 & 66,6 & 0,436 \\
\hline Limitações AVDs & 51,4 & 33,3 & 21,9 & 16,6 & 0,045 \\
\hline Limitações físicas & 37,8 & 33,3 & 15,1 & 0 & 0,023 \\
\hline Limitações sociais & 36,8 & 33,3 & 14,1 & 11,1 & 0,008 \\
\hline Relações pessoais & 0 & 0 & 0 & 0 & 1,000 \\
\hline Emoções & 32,2 & 22,2 & 10 & 0 & 0,028 \\
\hline Sono e disposição & 37,8 & 33,3 & 19,6 & 16,6 & 0,109 \\
\hline Medidas de gravidade & 42,3 & 46,6 & 24,2 & 26,6 & 0,005 \\
\hline Escala de sintomas & 10,7 & 11 & 5,7 & 4 & 0,016 \\
\hline
\end{tabular}

\section{DISCUSSÃO}

A incontinência urinária determina problemas econômicos, físicos, sociais e psicológicos, alterando de forma importante a saúde da mulher. A terapêutica conservadora para a IU, utilizando recursos fisioterapêuticos, pode reforçar o controle esfincteriano através do fortalecimento da musculatura do assoalho pélvico, reduzindo assim os sintomas da perda urinária e consequentemente a melhora da qualidade de vida.

Ao avaliar os fatores de risco para a IU, um estudo constatou que o parto vaginal está associado com o aumento de casos de IU quando comparado com o parto cesáreo; no entanto, o parto vaginal isoladamente não é o causador da IU e sim, quando associado às lesões, traumas do assoalho pélvico e a outros fatores de risco. Tais achados coincidem com nossa amostra, onde foi observada uma média de 5,09 no número de partos normais. ${ }^{22}$

No presente estudo, a maioria das pacientes (6) foi acometida por IUM, baseado nas queixas clínicas, assim como no estudo que, ao correlacionar a queixa clínica com os achados urodinâmicos de 114 mulheres portadoras de IU, 
constataram que $52,6 \%$, de acordo com a queixa clínica, eram portadoras de IUM. ${ }^{23}$ Observa-se como uma das limitações deste estudo a falta de dados de exame urodinâmico para uma avaliação mais precisa, visto que as pacientes eram portadoras de diferentes tipos de IU.

Como já descrito em pesquisas, o desconhecimento sobre como funciona a musculatura do assoalho pélvico pode ser um fator precipitante para IU, pois o desconhecimento da função muscular pode levar a uma hipotrofia e fraqueza. Esse dado foi encontrado em 100\% das participantes do nosso estudo. ${ }^{24}$

No presente estudo, observou-se melhora acentuada das pacientes, na perda de urina diária e no alívio dos sinais e sintomas referidos, onde a média da frequência de micções noturnas na presença de noctúria e do número de situações de perda urinária aos esforços foi menor que a anterior ao tratamento com a cinesioterapia. Houve ainda redução no número de pacientes com dificuldade de retenção e perda involuntária de urina ao primeiro desejo forte de urinar e no número de absorventes diários utilizados.

Os resultados obtidos neste estudo estão concordes com outros estudos que demonstram melhora significativa dos sinais e sintomas da IU em mulheres incontinentes quando submetidas a tratamento conservador através da cinesioterapia do assoalho pélvico. ${ }^{25-27}$

Segundo Mouritsen, estudos mostraram conclusivamente que os exercícios do assoalho pélvico têm melhor prognóstico em casos de incontinência moderada e de curta duração. Pacientes mais jovens, que são pré-menopáusicas ou recebendo estrógenos, com peso normal e sem cirurgias prévias para incontinência, e que tenham demonstrado alguma conscientização e função de sua musculatura do assoalho pélvico, têm o melhor prognóstico. ${ }^{28}$ Contudo, nesta pesquisa observou-se boa resposta ao tratamento, tanto nas pacientes que desenvolveram os sintomas entre um a três anos quanto nas pacientes que notaram os primeiros sintomas há mais de dez anos.
O impacto que a incontinência causa na vida social provoca restrições quanto a frequentar lugares públicos, viajar, dormir fora de casa e até fazer visitas aos amigos. Isto está relacionado ao fato de as mulheres evitarem sair de casa, pois além de ficarem envergonhadas e com medo de cheirarem a urina, não sabem se encontrarão um local adequado para realizar suas micções e sua higiene pessoa. ${ }^{10}$

A partir da análise do questionário de qualidade de vida Kings Health Questionnaire (KHQ) nas pacientes estudadas, foi possível observar uma melhora aparentemente significativa da qualidade de vida no pós tratamento com a cinesioterapia do assoalho pélvico. Corroborando nosso estudo, o autor, ao avaliar qualidade de vida de mulheres após tratamento da IUE com fisioterapia, observou melhora significativa da qualidade de vida dessas mulheres. ${ }^{29}$

Outra pesquisa enfatiza a importância do impacto prejudicial da IU na qualidade de vida e afirma ainda que apenas a depressão tem impacto maior quando comparada a pacientes portadores de diabetes, hipertensão ou IU..$^{30}$

Assim, este estudo vem confirmar que a cinesioterapia do assoalho pélvico, como tratamento conservador para incontinência urinária em mulheres idosas, é um método efetivo, seguro e de baixo custo, que contribui para ampliar as possibilidades terapêuticas desta enfermidade.

\section{CONCLUSÃO}

Com os resultados obtidos, pode-se concluir que a cinesioterapia do assoalho pélvico foi positiva para obter melhoras reais e significativas sobre a perda de urina diária e o alívio dos sinais e sintomas referidos, bem como na qualidade de vida das idosas portadoras de IU.

Apesar das dificuldades presentes neste estudo, como o baixo número de mulheres estudadas, a dificuldade de aderência ao tratamento até o término do estudo, visto o número alto de exclusões por abandono ao tratamento, falta de equipamentos para 
uma avaliação mais fidedigna e resultados mais precisos para os diferentes tipos de IU encontradas, constatou-se melhora aparentemente significante do quadro de perda urinária diária e alívio dos sinais e sintomas, usando como protocolo de tratamento a cinesioterapia do assoalho pélvico.

\section{REFERÊNCIAS}

1. Abrams P, Cardoso Fall M, Griffiths D, Rosier P, Ulmsten U, Van Kerrebroeck P, Victor A, Wein $\mathrm{A}$. The standartization of terminology of lower urinary tract: report from the standartization subcommitee of the International continence Society. Neurourol Urodyn 2002; 21(2):167-178.

2. Barracho ELLS, Dias RC, Saleme CS, Laranjeira CLS, Lima RSBC. Impacto sobre a quantidade de urina perdida de uma intervenção fisioterapêutica em idosas com incontinência urinária. Fisioterapia e Pesquisa 2006;13(1): 23-29.

3. Reis RB, Cologna AJ, Martins ACP, Paschoalin EL, Tucci E, Suaid HJ. Incontinência urinária no idoso. Acta Cirúrg Bras 2003;18(5): 47-51.

4. Honório MO, Santos SMA. Incontinência urinária e envelhecimento: impacto no cotidiano e na qualidade de vida. Rev Bras Enferm 2009; 62(1): 51-56.

5. Hermann V, Potrick BA, Palma PCR, et al. Eletroestimulação transvaginal do assoalho pélvico no tratamento da incontinência urinária de esforço: avaliações clínica e ultra sonográfica. Rev Assoc Méd Brás 2003;49 (4): 401-5.

6. Bandeira EMFS, Pimenta FAP, Souza MC. Atenção à saúde do idoso. .Belo Horizonet: Secretaria de Estado de Saúde de Minas Gerais; 2006.

7. Varmus H. Disease-specific estimates of direct and indirect costs of illness and $\mathrm{NIH}$ support. Washington :Bethesda ;1997.

8. Subak LS, Brown JS, Kraus SR, Brubaker L, Lin F, Richter HE, Bradley CS, Grady . Diagnostic aspects of incontinence study (DAISy) Group : The "Costs" of urinary incontinence for women. Obstet Gynecol 2006 april ; $107(4)$ : 908916.

9. Moreira $\mathrm{ECH}$,et al. Tratamento cirúrgico e conservador da incontinência urinária de esforço. Fisioterapia em Movimento 2001;13(2):9-13.
Assim, programas com essas características têm possibilidade de serem difundidos e podem ser facilmente implementados em locais menos convencionais, como possibilidades terapêuticas menos onerosas de atenção à saúde da mulher, garantindo-lhes melhor qualidade de vida.

10. Lopes MHBM, Higa R. Restrições causadas pela incontinência urinária à vida da mulher. Rev. Esc Enferm 2006; 40( 1): 34-41.

11. Belo J, Francisco E, Leite H, Catarino A. Reeducação do pavimento pélvico com cones de plevnik em mulheres com incontinência urinária. Acta Méd Port 2005;18: 117-122.

12. Grosse D, Sengler J. Reeducação Perineal. São Paulo: Manole; 2002.

13. Seleme RS. Incontinência urinária: um problema social de saúde pública. . Rio de Janeiro. Tese (Tese em Serviço Social) Universidade Federal do Rio de Janeiro; 2006.

14. Borges FD, Frare JC, Moreira ECH. Fisioterapia na Incontinência Urinária. Fisioterapia em Movimento 1998 mar; 10(2): 103111.

15. Palma PCR, Ricceto CLZ. Incontinência urinária na mulher In: Brata HS, Carvalhal GF. Urologia : princípios e prática. Porto Alegre: Artmed; 1999. p. 209 - 216.

16. Freitas $\mathrm{F}$, Menke $\mathrm{CH}$, Rrivoire W. Rotinas em ginecologia. 4. ed. Porto Alegre: Artmed; 2002.

17. Kegel AH. Physiologic therapy for urinary stress incontinence. Jama 1951; 163(10): 915-17.

18. Kegel A, Powell TO. The physiological treatment of urinary stress incontinence. J Urolog 1950; 63(5): 808-13.

19. Wroclawski ER, Borrelli JRM, Borrelli M. Tratamento não-Cirúrgico da incontinência urinária de esforço. In: Rubinstein I. Urologia feminina. São Paulo: BYK; 1999. p. 189 - 198.

20. Tamanini JTN, $\mathrm{D}^{\prime}$ ancona CAL, Botega NJ, Rodrigues NNJ. Validação do "King's Health Questionnaire" para o português em mulheres com incontinência urinária. Rev Saúde Pública 2003; 37(2) : 203-11.

21. Siegel S, Castellan Jr NJ. Estatística não paramétrica para ciências do comportamento. 2 ed. Porto Alegre 2006: Artmed. p.448. 
22. Higa R, Lopes MHBM, Reis MJ. Fatores de risco para a incontinência urinária na mulher. Rev. Esc. Enferm. 2008; 42(1): 187-92.

23. Feldner Jr PC, Bezerra LRPS, Girão MJBC, Castro RA, Sartori MGF, Baracat EC, Lima GR. Valor da queixa clínica e exame físico no diagnóstico da incontinência urinária. Rev. Bras Ginecol Obstetr 2002; 24(2): 87-9.

24. Leon MIWH. A eficácia de um programa cinesioterapêutico para mulheres idosas com incontinência urinária. Fisioterapia Brasil 2001; 2(2): 107-15.

25. Marques SMF, Freitas PAC. A cinesioterapia como tratamento da incontinência urinária na unidade básica de saúde. Fisioterapia em Movimento 2005; 18(4). p. 63-67.

26. Bernardes N, Péres F, Souza E, et al. Métodos de tratamento utilizados na incontinência urinária de esforço genuína: um estudo comparativo entre Cinesioterapia e Eletroestimulação Endovaginal. Rev Bras Ginecol Obstetr 2000; 22(1): 281-90.

27. Zacchi AS, Bruse CF, Souza RMN. Utilização da cinesioterapia na incontinência urinária de esforço. Fisio Terapia 2008 set/out; 5(28) : 10-11.

28. Mouritsen L. Pelvic Floor: exercises for female stress urinary incontinence. The International Urogynecology Journal 1994; 34(5) : 44 - 51.

29. Rett MT, Simões JA, Herrmann V, Gurgel MSC, Morais SS. Qualidade de vida em mulheres após tratamento da incontinência urinária de esforço com fisioterapia. Rev Bras Ginecol Obstetr 2007; 29(3):134-40.

30. Chen GD, Lin TL, Hu SW ,et al.. Prevalence and correlation of urinary incontinence and overactive bladder in taiwanese women. Neurourol Urodyn 2003; 22(2): 109-17. 
